\title{
Recombinant CTAP III
}

National Cancer Institute

\section{Source}

National Cancer Institute. Recombinant CTAP III. NCI Thesaurus. Code C1358.

Connective Tissue-Activating Peptide III is a platelet-derived growth factor that stimulates in fibroblasts mitogenesis, extracellular matrix synthesis, glucose metabolism, and plasminogen activator synthesis through interaction with a GPCR. It is present in platelet granules, released by inducers of platelet aggregation, and is chemoattractant for fibroblasts, presumably for repair. Pro-platelet basic protein is the precursor of platelet basic protein (PBP) and CTAP III. Upon platelet activation they are released and processed in plasma to beta-thrombog lobulin and neutrophil-activating peptide-2. The thrombocidins TC1 and TC2 are variants of NAP2 and CTAP III, respectively. TC1 and TC2 differ from NAP2 and CTAP III by their bactericidal and fungicidal properties, which apparently do not involve pore formation. (from OMIM 121010 and $\mathrm{NCI}$ ) 